\title{
Quasiparticle properties of an impurity in a Fermi gas
}

\author{
Jonas Vlietinck, Jan Ryckebusch, and Kris Van Houcke* \\ Department of Physics and Astronomy, Ghent University, Proeftuinstraat 86, 9000 Gent, Belgium
}

(Received 7 January 2013; published 25 March 2013)

\begin{abstract}
We report a study of a spin-down impurity strongly coupled to a spin-up Fermi sea (a so-called Fermi polaron) with the diagrammatic Monte Carlo (DiagMC) technique. Conditions of zero temperature and three dimensions are considered for an ultracold atomic gas with resonant interactions in the zero-range limit. A Feynman diagrammatic series is developed for the one-body and two-body propagators providing information about the polaron and molecule channel, respectively. The DiagMC technique allows us to reach diagram orders that are high enough for extrapolation to infinite order. The robustness of the extracted results is examined by checking various resummation techniques and by running the simulations with various choices for the propagators and vertex functions. It turns out that dressing the lines in the diagrams as much as possible is not always the optimal choice. We also identify classes of dominant diagrams for the one-body and two-body self-energy in the region of strong interaction. These dominant diagrams turn out to be the leading processes of the strong-coupling limit. The quasiparticle energies and $Z$ factor are obtained as a function of the interaction strength. We find that the DiagMC results for the molecule and polaron properties are very similar to those obtained with a variational ansatz. Surprisingly, this variational ansatz gives very good predictions for the quasiparticle residue even when this residue is significantly less than 1 .
\end{abstract}

DOI: 10.1103/PhysRevB.87.115133

PACS number(s): 05.30.Fk, 03.75.Ss, 02.70.Ss

\section{INTRODUCTION}

The notion of a "bare" particle loses its significance once it is strongly coupled to a medium. Landau introduced the notion of a quasiparticle whose properties may be very different from those of a bare particle. ${ }^{1}$ The most prominent example is an electron moving in a crystal: the electron displaces the nearby ions and carries this distortion with it. The presence of the phonon cloud changes the mass and energy of the electron, which is dubbed a "polaron." 2 More generally, a polaron arises whenever a quantum impurity is strongly coupled to an environment. These quantum-mechanical quasiparticles play a key role in the low-energy behavior of a macroscopic quantum liquid.

In recent years, the field of ultracold atoms has provided an exciting framework for studying polaronic effects. A key idea is that models designed for describing the rich and nontrivial structure of the solid state can be emulated in a clean and controllable manner with ultracold atoms. For example, so-called Fermi polarons, ${ }^{3-5}$ spin-down impurities that are strongly coupled to a spin-up Fermi sea (FS), can be created in a degenerate two-component atomic Fermi gas when going to the limit of strong spin imbalance close to a Feshbach resonance. The impurity is coherently dressed with particle-hole excitations of the FS. The properties of the Fermi polaron are important for the quantitative understanding of a strongly imbalanced Fermi gas. ${ }^{6}$

In this paper, we focus on the "attractive Fermi polaron," with an attractive interaction between the impurity and the fermions of the bath. A recent experiment using an ultracold gas of ${ }^{6} \mathrm{Li}$ atoms in three dimensions revealed the existence of Fermi polarons through a narrow quasiparticle peak in the impurities' radio-frequency spectrum. ${ }^{4}$ At a critical interaction strength, the disappearance of this peak was interpreted as a transition from polaronic to molecular binding, when the impurity and an atom of the sea form a two-body bound state. Such a transition had theoretically been predicted in three dimensions by Prokof'ev and Svistunov. ${ }^{3}$ To determine the transition point, they developed a diagrammatic Monte Carlo technique (DiagMC) capable of solving the Fermi polaron model. ${ }^{3,8}$ Calculations of the ground-state energy showed that for a sufficiently strong attraction between the impurity atom and the atoms of the spin-up FS, a molecular state becomes energetically favorable. The crossing point was found at an interaction strength $\left(k_{F} a\right)_{c}=1.11(2)$, with $k_{F}$ the Fermi momentum of the spin-up sea and $a$ the $s$-wave scattering length. A variational treatment developed by Chevy based on an expansion up to single particle-hole excitations on top of the unperturbed FS turned out to be remarkably accurate. ${ }^{9}$ A combination of Chevy's ansatz with a variational wave function in the molecular limit ${ }^{10-12}$ also revealed the polaron-to-molecule transition, very close to the DiagMC result.

In the present work, we study the quasiparticle properties of the Fermi polaron problem in three dimensions with the DiagMC technique. ${ }^{3,8}$ This technique evaluates a series of Feynman diagrams for the one-particle and two-particle proper self-energies. A full description of the DiagMC algorithm was presented in Ref. 8. Building on the work in Ref. 8 we have implemented the DiagMC algorithm independently. We explore various DiagMC schemes ${ }^{13}$ and series resummation methods to check the robustness of the results against the possible uncertainties of summing the series. First, we confirm the transition point. Next, we calculate the quasiparticle residue, which we compare to experimental data and variational results. The quasiparticle residue, or $Z$ factor, gives the overlap of the noninteracting wave function and the fully interacting one,

$$
Z_{p}=\left|\left\langle\Psi_{0}^{N_{\uparrow}} \mid \mathbf{0}_{\downarrow}, \operatorname{FS}\left(N_{\uparrow}\right)\right\rangle\right|^{2}
$$

with $\left|\Psi_{0}^{N_{\uparrow}}\right\rangle$ the fully interacting ground state and $\left|\mathbf{0}_{\downarrow}, \operatorname{FS}\left(N_{\uparrow}\right)\right\rangle$ a free spin-down atom carrying momentum $\mathbf{p}=\mathbf{0}$ in a noninteracting FS of $N_{\uparrow}$ spin-up atoms. The spin-up atoms 
are noninteracting since $p$-wave scattering is negligible. The residue reflects the impurity's probability of free propagation.

The outline of the paper is as follows. In Sec. II we introduce the model and the structure of the Feynman diagrammatic expansion. In Sec. III we discuss the results of the numerical calculations. Thereby, we investigate how the results depend on the choices made with regard to the diagrammatic series, like the use of bare versus dressed propagators. Also, the resummation of the diagrammatic series is discussed in depth. The results for the quasiparticle properties, like the residue, are the subject of Sec. IV.

\section{MODEL AND DIAGRAMMATIC STRUCTURE}

We consider a dilute two-component gas of ultra-cold fermionic atoms interacting via the van der Waals potential. The Hamiltonian has a kinetic and interaction term,

$$
\begin{aligned}
\hat{H}= & \sum_{\mathbf{k}, \sigma=\uparrow \downarrow} \epsilon_{\mathbf{k} \sigma} \hat{c}_{\mathbf{k} \sigma}^{\dagger} \hat{c}_{\mathbf{k} \sigma} \\
& +\frac{1}{\mathcal{V}} \sum_{\mathbf{k}, \mathbf{k}^{\prime}, \mathbf{q}} V\left(\mathbf{k}-\mathbf{k}^{\prime}\right) \hat{c}_{\mathbf{k}+\frac{\mathbf{q}}{2} \uparrow}^{\dagger} \hat{c}_{-\mathbf{k}+\frac{\mathbf{q}}{2} \downarrow}^{\dagger} \hat{c}_{-\mathbf{k}^{\prime}+\frac{\mathbf{q}}{2} \downarrow} \hat{c}_{\mathbf{k}^{\prime}+\frac{\mathbf{q}}{2} \uparrow} .
\end{aligned}
$$

The operators $\hat{c}_{\mathbf{k} \sigma}^{\dagger}\left(\hat{c}_{\mathbf{k} \sigma}\right)$ create (annihilate) fermions with momentum $\mathbf{k}$ and spin $\sigma$. The spin- $\sigma$ fermions have mass $m_{\sigma}$ and dispersion $\epsilon_{\mathbf{k} \sigma}=k^{2} / 2 m_{\sigma}$, and $\mathcal{V}$ is the volume of the system. We take $\hbar=1$ throughout the paper and consider the mass-balanced case $m_{\uparrow}=m_{\downarrow}=m$. All the theoretical considerations are for zero temperature (or $T \ll T_{F}$, with $T_{F}$ the Fermi temperature). The diluteness of the system ensures that the range $b$ of the potential is much smaller than the typical interparticle distance $1 / k_{F}$, or $k_{F} b \ll 1$, with $k_{F}$ the Fermi momentum of the spin-up sea, and therefore the details of the interaction potential become irrelevant. Accordingly, without loss of generality, one can model the short-ranged interaction as a contact interaction, $V(\mathbf{r})=g_{0} \delta(\mathbf{r})$, in combination with the standard ultraviolet divergence regularization procedure described below.

The one-body and two-body propagators provide access to information about the "polaron" and "molecule" channel, respectively. The polaron and molecule are two distinct objects belonging to different charge sectors. The one-body and two-body propagators are discussed in Secs. II A and IIC. The adopted regularization procedure for the renormalized interaction is the subject of Sec. II B. The DiagMC method is introduced in Sec. II D.

\section{A. One-body propagator}

The polaron quasiparticle properties can be extracted from the impurity's Green's function, defined as

$$
G_{\downarrow}(\mathbf{k}, \tau)=-\theta(\tau)\left\langle\Phi_{0}^{N_{\uparrow}}\left|\hat{c}_{\mathbf{k} \downarrow}(\tau) \hat{c}_{\mathbf{k} \downarrow}^{\dagger}(0)\right| \Phi_{0}^{N_{\uparrow}}\right\rangle,
$$

with $\hat{c}_{\mathbf{k} \downarrow}(\tau)$ the annihilation operator in the Heisenberg picture,

$$
\hat{c}_{\mathbf{k} \downarrow}(\tau)=e^{\left(\hat{H}-\mu \hat{N}_{\downarrow}-\mu_{\uparrow} \hat{N}_{\uparrow}\right) \tau} \hat{c}_{\mathbf{k} \downarrow} e^{-\left(\hat{H}-\mu \hat{N}_{\downarrow}-\mu_{\uparrow} \hat{N}_{\uparrow}\right) \tau} .
$$

The propagator $G_{\downarrow}(\mathbf{k}, \tau)$ is written in the momentum imaginary-time representation, $\mu$ is a free parameter, $\hat{N}_{\sigma}$ is the number operator for spin- $\sigma$ particles, and $\mu_{\uparrow}$ is the chemical potential of the spin-up sea. The state

$$
\left|\Phi_{0}^{N_{\uparrow}}\right\rangle=|\rangle_{\downarrow}\left|\operatorname{FS}\left(N_{\uparrow}\right)\right\rangle
$$

consists of the spin-down vacuum and the noninteracting spinup FS. Since we are dealing with an impurity spin-down atom, $G_{\downarrow}$ is only nonzero for times $\tau>0$. The ground-state energy and $Z$ factor can be extracted from the Green's function of Eq. (3). Inserting a complete set of eigenstates $\left|\Psi_{n}^{N_{\uparrow}}\right\rangle$ of the full Hamiltonian, (2), for one spin-down particle and $N_{\uparrow}$ spin-up particles into Eq. (3) yields, for $\mathbf{k}=\mathbf{0}$,

$$
\begin{aligned}
G_{\downarrow}(\mathbf{0}, \tau)= & \theta(\tau) \sum_{n}\left|\left\langle\Psi_{n}^{N_{\uparrow}}\left|\hat{c}_{\mathbf{0} \downarrow}^{\dagger}\right| \Phi_{0}^{N_{\uparrow}}\right\rangle\right|^{2} \\
& \times e^{-\left(E_{n}\left(N_{\uparrow}\right)-E_{\mathrm{FS}}-\mu\right) \tau} \\
\stackrel{\tau \rightarrow+\infty}{=} & -Z_{p} e^{-\left(E_{p}-\mu\right) \tau},
\end{aligned}
$$

with $E_{p}$ the energy of the polaron, $E_{n}\left(N_{\uparrow}\right)$ the energy eigenvalues of Hamiltonian (2), and $E_{\mathrm{FS}}=3 \epsilon_{F} N_{\uparrow} / 5$ the energy of the ideal spin-up Fermi gas, with $\epsilon_{F}=k_{F}^{2} /(2 m)$ the Fermi energy.

The difference between the polaronic and the molecular state is embedded in the factors $\left|\left\langle\Psi_{n}^{N_{\uparrow}}\left|\hat{c}_{\mathbf{0} \downarrow}^{\dagger}\right| \Phi_{0}^{N_{\uparrow}}\right\rangle\right|^{2}$ in Eq. (6). For situations where the polaron is a well-defined quasiparticle in the ground state $\left|\Psi_{0}^{N_{\uparrow}}\right\rangle$, we have Eq. (1) for the $Z$ factor and $E_{p}=E_{0}\left(N_{\uparrow}\right)-E_{\mathrm{FS}}$. If, on the other hand, the ground state $\left|\Psi_{0}^{N_{\uparrow}}\right\rangle$ is a dressed molecule, the overlap $\left\langle\Psi_{0}^{N_{\uparrow}}\left|\hat{c}_{\mathbf{0} \downarrow}^{\dagger}\right| \Phi_{0}^{N_{\uparrow}}\right\rangle$ is $0 .{ }^{11}$ This is clear from the expansion of the molecular state in the number of particle-hole excitations:

$$
\begin{aligned}
\left|\Psi_{0}^{N_{\uparrow}}\right\rangle= & \left(\sum_{\mathbf{k}}^{\prime} \xi_{\mathbf{k}} \hat{c}_{-\mathbf{k} \downarrow}^{\dagger} \hat{c}_{\mathbf{k} \uparrow}^{\dagger}\right. \\
& \left.+\sum_{\mathbf{k}, \mathbf{k}^{\prime}, \mathbf{q}}^{\prime} \xi_{\mathbf{k} \mathbf{k}^{\prime} \mathbf{q}} \hat{c}_{\mathbf{q}-\mathbf{k}-\mathbf{k}^{\prime} \downarrow}^{\dagger} \hat{c}_{\mathbf{k} \uparrow}^{\dagger} \hat{c}_{\mathbf{k}^{\prime} \uparrow}^{\dagger} \hat{c}_{\mathbf{q} \uparrow}+\cdots\right)\left|\Phi_{0}^{N_{\uparrow}-1}\right\rangle .
\end{aligned}
$$

The coefficients $\xi$ are variational parameters, and the primes indicate that the sums on $\mathbf{k}, \mathbf{k}^{\prime}$, and $\mathbf{q}$ are restricted to $|\mathbf{k}|,\left|\mathbf{k}^{\prime}\right|>$ $k_{F}$ and $|\mathbf{q}|<k_{F}$. Even if a molecule is formed in the ground state, the polaron can be a well-defined excited state (in the sense of a narrow peak in the spectral function), and $Z_{p}$ can be nonzero.

For vanishing interactions $V$ the impurity Green's function of Eq. (3) becomes

$$
G_{\downarrow}^{0}(\mathbf{k}, \tau)=-\theta(\tau) e^{-\left(\epsilon_{\mathbf{k} \downarrow}-\mu\right) \tau} .
$$

The one-body propagator for the spin-up sea is defined as

$$
G_{\uparrow}(\mathbf{k}, \tau)=-\left\langle\Psi_{0}^{N_{\uparrow}}\left|T_{\tau}\left[\hat{c}_{\mathbf{k} \uparrow}(\tau) \hat{c}_{\mathbf{k} \uparrow}^{\dagger}(0)\right]\right| \Psi_{0}^{N_{\uparrow}}\right\rangle,
$$

with $T_{\tau}$ the time-ordering operator. Without interactions, one obtains the free propagator

$$
G_{\uparrow}^{0}(\mathbf{k}, \tau)=\left\{\begin{array}{lll}
-e^{-\left(\epsilon_{\mathbf{k}}-\epsilon_{F}\right) \tau} \theta\left(|\mathbf{k}|-k_{F}\right) & \text { if } & \tau>0 ; \\
e^{-\left(\epsilon_{\mathbf{k}}-\epsilon_{F}\right) \tau} \theta\left(k_{F}-|\mathbf{k}|\right) & \text { if } & \tau<0 .
\end{array}\right.
$$

Our goal is to calculate the $G_{\downarrow}$ of Eq. (3) to extract $E_{p}$ by means of Eq. (6). This is achieved by summing all irreducible one-particle self-energy diagrams with the DiagMC algorithm (which works in the momentum-imaginary-time representation). The irreducible self-energy $\Sigma(\mathbf{k}, \omega)$ in the imaginaryfrequency representation is obtained after a numerical Fourier 


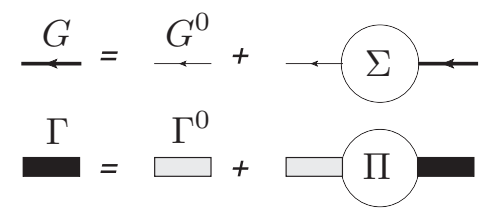

FIG. 1. Graphical representation of the Dyson equation and the Bethe-Salpeter equation. The free (dressed) one-body impurity propagator is denoted $G_{\downarrow}^{0}\left(G_{\downarrow}\right)$. $\Sigma$ and $\Pi$ are the one-body and two-body self-energies, respectively. $\Gamma$ is the fully dressed interaction, wheres $\Gamma^{0}$ is the partially dressed interaction, obtained by summing all the bare ladders $G_{\downarrow}^{0} G_{\uparrow}^{0}$ [see Eqs. (16) and (17)].

transform and inserted into Dyson's equation to give $G_{\downarrow}$,

$$
\left[G_{\downarrow}(\mathbf{k}, \omega)\right]^{-1}=\left[G_{\downarrow}^{0}(\mathbf{k}, \omega)\right]^{-1}-\Sigma(\mathbf{k}, \omega),
$$

with $\omega$ the imaginary frequency. A graphical representation of the Dyson equation is shown in the top panel in Fig. 1.As shown in Ref. 8, the polaron energy $E_{p}$ and $Z$ factor $Z_{p}$ can be extracted directly from the self-energy $\Sigma(\mathbf{0}, \tau)$ :

$$
\begin{gathered}
E_{p}=\int_{0}^{+\infty} d \tau \Sigma(\mathbf{0}, \tau) e^{\left(E_{p}-\mu\right) \tau} ; \\
Z_{p}=\frac{1}{1-\int_{0}^{+\infty} d \tau \tau \Sigma(\mathbf{0}, \tau) e^{\left(E_{p}-\mu\right) \tau}} .
\end{gathered}
$$

The effective mass $m_{*}$ of the polaron is evaluated with the estimator $^{8}$

$$
m_{*}=\frac{1 / Z_{p}}{1 / m+B_{0}},
$$

with

$$
B_{0}=\int_{0}^{+\infty} d \tau e^{\left(E_{p}-\mu\right) \tau}\left[\left.\frac{1}{3} \nabla_{\mathbf{k}}^{2} \Sigma(\mathbf{k}, \tau)\right|_{k=0}\right],
$$

which can conveniently be estimated by expanding $\Sigma(\mathbf{k}, \tau)$ in Legendre polynomials. One obtains

$$
\left.\frac{1}{3} \nabla_{\mathbf{k}}^{2} \Sigma(\mathbf{k}, \tau)\right|_{k=0}=\frac{15}{2 \Delta^{3}} \int_{0}^{\Delta} d k \Sigma(k, \tau)\left(\frac{3 k^{2}}{\Delta^{2}}-1\right),
$$

and the integral can be evaluated during the MC simulation. The upper limit of integration $(\Delta)$ is optimized to minimize the statistical noise while avoiding a systematic error at too large $\Delta$. We also used an alternative way: calculating the quasiparticle spectrum $E(\mathbf{k})$ and fitting $m_{*}$ via $E(\mathbf{k})=$ $E_{p}+k^{2} /\left(2 m_{*}\right)$.

\section{B. Renormalized interaction}

We introduce the $s$-wave scattering length $a$ for collisions between spin-up and spin-down particles. One of the advantages of working with Feynman diagrams is that one can work directly in the zero-range limit $k_{F} b \rightarrow 0$ (or, equivalently, $\Lambda / k_{F} \rightarrow+\infty$, with $\Lambda$ the ultraviolet momentum cutoff) while keeping $k_{F} a$ constant. Thereby, the ultraviolet physics can be taken into account by means of a summation over all Feynman ladder diagrams.

In the momentum-imaginary-frequency representation $(\mathbf{p}, \Omega)$, one obtains for the partially dressed interaction

$$
\Gamma^{0}(\mathbf{p}, \Omega)=g_{0}+g_{0} \Pi^{0}(\mathbf{p}, \Omega) \Gamma^{0}(\mathbf{p}, \Omega),
$$

with $\Pi^{0}$ the two-particle self-energy consisting of one "bare" ladder,

$$
\begin{aligned}
\Pi^{0}(\mathbf{p}, \Omega)= & -\frac{1}{2 \pi \mathcal{V}} \sum_{|\mathbf{q}|<\Lambda} \int d \omega G_{\uparrow}^{0}\left(\frac{\mathbf{p}}{2}+\mathbf{q}, \omega\right) \\
& \times G_{\downarrow}^{0}\left(\frac{\mathbf{p}}{2}-\mathbf{q}, \Omega-\omega\right) \\
= & \frac{1}{\mathcal{V}} \sum_{|\mathbf{q}|<\Lambda} \frac{\theta\left(|\mathbf{p} / 2+\mathbf{q}|-k_{F}\right)}{i \Omega-p^{2} /(4 m)-q^{2} / m+\mu+\epsilon_{F}},
\end{aligned}
$$

where the momentum cutoff $\Lambda$ is required to keep the sum finite. The bare coupling constant $V(\mathbf{p})=\int d \mathbf{r} e^{-i \mathbf{p} \cdot \mathbf{r}} V(\mathbf{r})=$ $g_{0}$ can be eliminated in favor of the physical scattering length $a$ by using standard scattering theory,

$$
\frac{1}{g_{0}}=\frac{m}{4 \pi a}-\frac{1}{\mathcal{V}} \sum_{|\mathbf{k}|<\Lambda} \frac{1}{2 \epsilon_{\mathbf{k}}} .
$$

The $\Gamma^{0}(\mathbf{p}, \Omega)$ from Eq. (16) can be expressed in terms of the $s$-wave scattering length $a$, by taking the limit $\Lambda \rightarrow+\infty$ and $g_{0} \rightarrow 0^{-}$with $a$ fixed. In this zero-range limit, one gets

$$
\left[\Gamma^{0}(\mathbf{p}, \Omega)\right]^{-1}=\left[\tilde{\Gamma}^{0}(\mathbf{p}, \Omega)\right]^{-1}-\bar{\Pi}(\mathbf{p}, \Omega),
$$

with

$$
\begin{aligned}
\bar{\Pi}(\mathbf{p}, \Omega)= & -\int \frac{d \mathbf{q}}{(2 \pi)^{3}} \theta\left(k_{F}-q\right) \\
& \times \frac{1}{i \Omega-\frac{q^{2}}{2 m}-\frac{(\mathbf{p}-\mathbf{q})^{2}}{2 m}+\mu+\epsilon_{F}} .
\end{aligned}
$$

Here, we have taken the thermodynamic limit $(\mathcal{V} \rightarrow+\infty$ and $N_{\uparrow} / \mathcal{V}$ fixed). The integral in Eq. (20) can be evaluated analytically, and the dressed interaction in vacuum is given by

$$
\left[\tilde{\Gamma}^{0}(\mathbf{p}, \Omega)\right]^{-1}=\frac{m}{4 \pi a}-\frac{m}{8 \pi} \sqrt{p^{2}-4 m\left(i \Omega+\mu+\epsilon_{F}\right)}
$$

for $\Omega \neq 0$ or $\mu<-\epsilon_{F}$, and assuming the principal branch. For $\mu<-\left[\epsilon_{F}+1 /\left(m a^{2}\right)\right]$, the Fourier transform to imaginary time can be done analytically, producing

$$
\begin{aligned}
\tilde{\Gamma}^{0}(\mathbf{p}, \tau)= & -\frac{4 \pi}{m^{3 / 2}} e^{-\left(\frac{p^{2}}{4 m}-\mu-\epsilon_{F}\right) \tau} \\
& \times\left(\frac{1}{\sqrt{\pi \tau}}+\frac{1}{\sqrt{m} a} e^{\frac{\tau}{m a^{2}}} \operatorname{erfc}\left(-\sqrt{\frac{\tau}{m}} \frac{1}{a}\right)\right),
\end{aligned}
$$

with $\operatorname{erfc}(x)$ the complementary error function. As in Ref. 8, we use $\Gamma^{0}(\mathbf{p}, \tau)$ as a partially dressed interaction vertex in the diagrammatic series, instead of the bare interaction vertex $g_{0}$. This dressed vertex is calculated here in an imaginary time representation by performing the Fourier transform of Eq. (19) numerically.

In the next step, the interaction vertex is fully dressed by calculating the two-particle self-energy $\Pi$ and plugging it into the Bethe-Salpeter equation,

$$
[\Gamma(\mathbf{p}, \Omega)]^{-1}=\left[\Gamma^{0}(\mathbf{p}, \Omega)\right]^{-1}-\Pi(\mathbf{p}, \Omega) .
$$

A graphical representation of this equation is shown in Fig. 1 . The self-energy $\Pi$ contains all connected two-particle diagrams that are irreducible with respect to cutting a single $\Gamma^{0}$ propagator. To avoid double counting, the diagrams for $\Pi$ should not contain any ladders, since these have been 
summed in $\Gamma^{0}$ by means of Eq. (16). This rule also holds when summing diagrams for the one-body self-energy $\Sigma$, built from free propagators $G_{\sigma}^{0}$ and $\Gamma^{0}$.

\section{Two-body propagator}

Here, we consider the pair annihilation operator,

$$
\hat{P}_{\mathbf{k}}=\sum_{\mathbf{q}} \varphi(\mathbf{q}) \hat{c}_{\mathbf{k}-\mathbf{q} \uparrow} \hat{c}_{\mathbf{q} \downarrow},
$$

with $\varphi(\mathbf{q})$ the momentum representation of the wave function $\varphi(\mathbf{r})$ for the relative motion of two fermions of opposite spin. The two-particle propagator is defined as

$$
G_{2}(\mathbf{k}, \tau)=-\theta(\tau)\left\langle\Phi_{0}^{N_{\uparrow}}\left|\hat{P}_{\mathbf{k}}(\tau) \hat{P}_{\mathbf{k}}^{\dagger}(0)\right| \Phi_{0}^{N_{\uparrow}}\right\rangle,
$$

where we have included the fact that the impurity spin- $\downarrow$ atom propagates forward in time. Inserting the complete basis $\left|\Psi_{n}^{N_{\uparrow}+1}\right\rangle$ for $\left(N_{\uparrow}+1\right)$ spin-up particles and one spin-down particle gives

$$
\begin{aligned}
G_{2}(\mathbf{0}, \tau)= & -\theta(\tau) \sum_{n}\left|\left\langle\Psi_{n}^{N_{\uparrow}+1}\left|\hat{P}_{\mathbf{0}}^{\dagger}\right| \Phi_{0}^{N_{\uparrow}}\right\rangle\right|^{2} \\
& \times e^{-\left(E_{n}\left(N_{\uparrow}+1\right)-E_{\mathrm{FS}}\left(N_{\uparrow}\right)-\mu_{\uparrow}-\mu\right) \tau} \\
\stackrel{\tau \rightarrow+\infty}{=} & -Z_{\mathrm{mol}} e^{-\left(E_{\mathrm{mol}}-\mu\right) \tau},
\end{aligned}
$$

with $E_{\text {mol }}$ the molecule energy and $Z_{\text {mol }}$ the molecule $Z$ factor. If the molecule is a well-defined quasiparticle in the ground state, we have

$$
Z_{\mathrm{mol}}=\left|\left\langle\Psi_{0}^{N_{\uparrow}+1}\left|\hat{P}_{\mathbf{0}}^{\dagger}\right| \Phi_{0}^{N_{\uparrow}}\right\rangle\right|^{2}
$$

and $E_{\mathrm{mol}}=E_{0}\left(N_{\uparrow}+1\right)-E_{\mathrm{FS}}\left(N_{\uparrow}\right)-\mu_{\uparrow}$. Note that the value of $Z_{\text {mol }}$ depends on the wave function $\varphi(\mathbf{q})$. The functional from of this pair wave function depends on the nature of the experiment used to probe the molecule.

In practice, it is easier to calculate the molecule energy from the fully dressed interaction $\Gamma$ [see Eq. (23)]. This function is closely related to the pair correlation function, namely,

$$
\Gamma(\mathbf{k}, \tau)=g_{0} \delta(\tau)+g_{0} \mathcal{P}(\mathbf{k}, \tau) g_{0},
$$

with

$$
\mathcal{P}(\mathbf{r}, \tau)=-\theta(\tau)\left\langle\Phi_{0}^{N_{\uparrow}}\left|\left(\hat{\Psi}_{\uparrow} \hat{\Psi}_{\downarrow}\right)(\mathbf{r}, \tau)\left(\hat{\Psi}_{\downarrow}^{\dagger} \hat{\Psi}_{\uparrow}^{\dagger}\right)(\mathbf{0}, 0)\right| \Phi_{0}^{N_{\uparrow}}\right\rangle
$$

the pair correlation function. The field operators $\hat{\Psi}_{\sigma}^{\dagger}(\mathbf{r})=$ $\sum_{\mathbf{k}} e^{-i \mathbf{k r}} \hat{c}_{\mathbf{k}, \sigma}^{\dagger} / \sqrt{\mathcal{V}}$ create a spin- $\sigma$ fermion at position $\mathbf{r}$. In Eq. (29) the pair of particles is created at the same position [which corresponds to $\varphi(\mathbf{q})=1$ in Eq. (24)]. The structure of the fully dressed interaction $\Gamma$ and the two-particle propagator $G_{2}$ now implies that both structures have the same poles [see Eq. (28)]. Therefore, the exponential tail of the function $\Gamma(\mathbf{k}=$ $\mathbf{0}, \tau$ ) can conveniently be used for estimating the molecule energy, rather than the tail of $G_{2}(\mathbf{0}, \tau)$. This is equivalent to looking for this pole of the Bethe-Salpeter equation, (23). The molecule's energy $E_{\mathrm{mol}}$ is given by the parameter $\mu$, which satisfies the equation

$$
\left[\Gamma^{0}(\mathbf{p}=\mathbf{0}, \Omega=0)\right]^{-1}=\Pi(\mathbf{p}=\mathbf{0}, \Omega=0),
$$
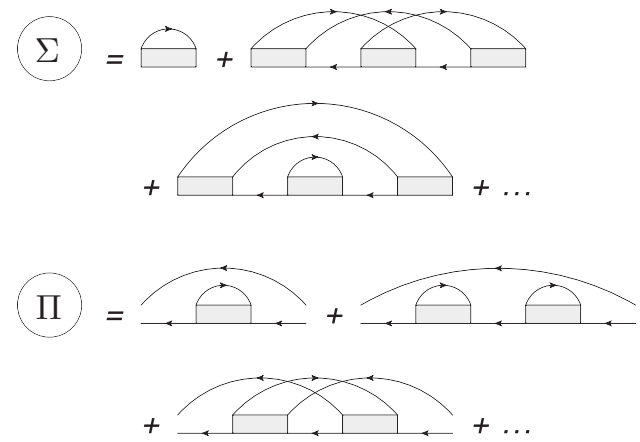

FIG. 2. Diagrammatic expansion for the one-body self-energy $\Sigma$ and the two-body self-energy $\Pi$. Here, the diagrams are built from the bare propagators $G_{\sigma}^{0}$ (thin lines) and the partially dressed interaction $\Gamma^{0}$ (gray box). All diagrams have a "backbone" structure, since we have a single impurity propagating forward in time and interacting with a Fermi sea of free particles.

where the left-hand side is known analytically and the righthand-side is evaluated with the DiagMC algorithm in the imaginary-time domain.

\section{Diagrammatic Monte Carlo}

The DiagMC evaluates the series of Feynman diagrams for the self-energy in a stochastic way. We deal with both one-body and two-body self-energies. In the first step, the self-energy is built from the free propagators $G_{\sigma}^{0}$ and the partially dressed interaction $\Gamma^{0}$ (obtained through summation of $G_{\downarrow}^{0} G_{\uparrow}^{0}$ ladders, as discussed in Sec. II B). We refer to this series as the "bare series." Figure 2 shows the one-body and two-body self-energy diagrams up to order 3 in the bare scheme. The order of a diagram is $N$ when there are $N$ dressed interactions $\Gamma$ (i.e., $N$ boxes) present in the $\Sigma$ diagram and $N-1$ boxes in the $\Pi$ diagram. Note that the diagrams cannot contain ladders since these have been taken into the vertex function $\Gamma^{0}$. To illustrate factorial growth with order, the number of one-body self-energy diagrams for given order $N \leqslant 12$ is given in the second column in Table I for the bare series.

TABLE I. Factorial increase in the number of Feynman diagrams. At fixed order $N$, the number of one-body self-energy diagrams is given for different types of series: the bare series, the skeleton series with dressed $G_{\downarrow}$ (bold $G$ ), and the skeleton series with dressed $G_{\downarrow}$ and $\Gamma$ lines (bold $G-\Gamma$ ).

\begin{tabular}{lrrr}
\hline \hline$N$ & Bare & Bold $G$ & Bold $G-\Gamma$ \\
\hline 1 & 1 & 1 & 1 \\
2 & 0 & 1 & 0 \\
3 & 2 & 2 & 1 \\
4 & 6 & 7 & 2 \\
5 & 34 & 34 & 13 \\
6 & 210 & 206 & 74 \\
7 & 1526 & 1476 & 544 \\
8 & 12558 & 12123 & 458 \\
9 & 115618 & 111866 & 41221 \\
10 & 1177170 & 1143554 & 421412 \\
11 & 13136102 & 12816572 & 422881 \\
12 & 159467022 & 156217782 & 5753440 \\
\hline \hline
\end{tabular}




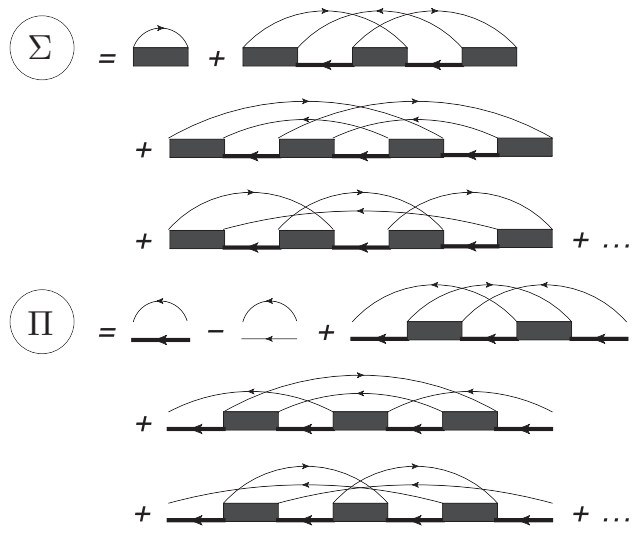

FIG. 3. Skeleton diagrammatic expansion for the one-body and two-body self-energies: the impurity propagator and interaction lines that appear in the diagram are fully dressed solutions of the Dyson equation and the Bethe-Salpeter equation (see Fig. 1).

In the second step, we use dressed propagators or "bold lines" in the diagrams. Such dressed (skeleton) series are evaluated with the bold DiagMC technique. ${ }^{3,13}$ We consider the case with only dressed $G_{\downarrow}$ propagators while keeping $\Gamma^{0}$ in Eqs. (19) and (20) as a renormalized interaction, and the case where both one-body propagators and interactions are dressed. We refer to these skeleton series as "bold $G^{\prime \prime}$ and "bold $G-\Gamma$," respectively. In the latter case, the bold DiagMC algorithm is constructed as follows: given the approximate one-body and two-body self-energies $\Sigma$ and $\Pi$, the Dyson and Bethe-Salpeter equations are solved to deliver the one-body propagator $G_{\downarrow}$ and the dressed interaction $\Gamma$ [see Eqs. (10) and (23)]. In the next step, these are used to dress the series for $\Sigma$ and $\Pi$, which are evaluated stochastically with DiagMC up to order $N_{*}$. This self-consistent cycle is repeated until convergence is reached. Figure 3 shows the skeleton (bold $G$ $\Gamma)$ series for the one- and two-body self-energies up to order 4. Evidently, when dressing the lines in the self-energies, one has to keep track of the two-particle reducibility and systematically avoid any double counting. This typically means that at any order $N$ the numbers in the second column in Table I ("Bare") are an upper limit of the number of diagrams in the third and fourth columns. At $N=2$ and $N=4$, however, the number of diagrams increases due to the fact that ladders should be included again once $G_{\downarrow}$ is bold. All the diagrams in Table I are summed explicitly during the (bold) DiagMC simulation.

\section{RESUMMATION AND BOLDIFICATION}

When considering a diagrammatic series, it is natural to ask whether there are dominant classes of diagrams. Identification of the dominant diagrams potentially allows one to make good approximations. To address this issue, we constructed a histogram counting how many times a certain topology is sampled. We consider the bare series first. It turns out that for the one-body self-energy, roughly half of the simulation time is spent on sampling two diagrams at each order. These two diagrams are shown in Fig. 4 for diagram order 6. To understand why these two diagrams are dominant at a fixed $N$, we use an argument first made by Hugenholtz. ${ }^{14}$ For a dilute spin-up gas, momentum integration inside the FS is

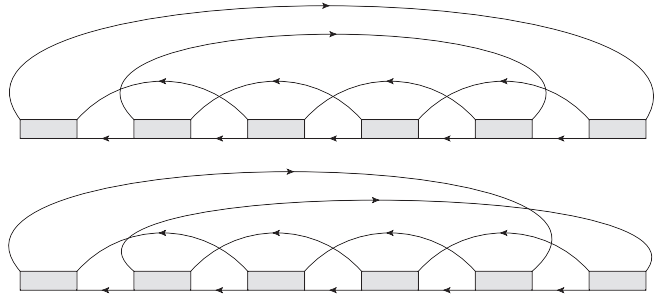

FIG. 4. The two dominant one-body self-energy diagrams for $N=6$. Imaginary time runs from right to left.

heavily restricted in phase space [momentum integration runs up to the Fermi momentum $\left.k_{F} \sim\left(N_{\uparrow} / \mathcal{V}\right)^{1 / 3}\right]$. This implies the presence of a backward (or hole) spin-up propagator, reducing the contribution of the diagram significantly, while the forward (particle) propagator enhances the contribution by roughly a factor $\int_{|\mathbf{k}|>k_{F}} d \mathbf{k}$. As a consequence, diagrams with the smallest possible number of hole propagators will be dominant. For the self-energy, we see that, at fixed order, the minimum number of hole propagators is 2 . Since the number of fermion loops differs by 1 , these two diagrams have opposite sign. Numerically we found that the two diagrams almost cancel each other. This can be seen in Fig. 5, where we show the polaron energy $E_{p}$ as a function of the inverse diagram order cutoff $N_{*}$ for the interaction strength $1 /\left(k_{F} a\right)=0$. For the "bare series," we observe a fast convergence due to cancellation of diagrams. This magic cancellation was referred to as "sign blessing." At infinite scattering length, such near-cancellation was also observed by Combescot and Giraud. ${ }^{15}$ They found that the success of the Chevy ansatz at strong coupling can be attributed to a nearly perfect destructive interference of the states with more than one particle-hole excitation. Combescot and Giraud illustrated that an expansion in powers of the hole wave vectors converges extremely rapidly at unitarity. In our case, the series is organized differently, but at fixed

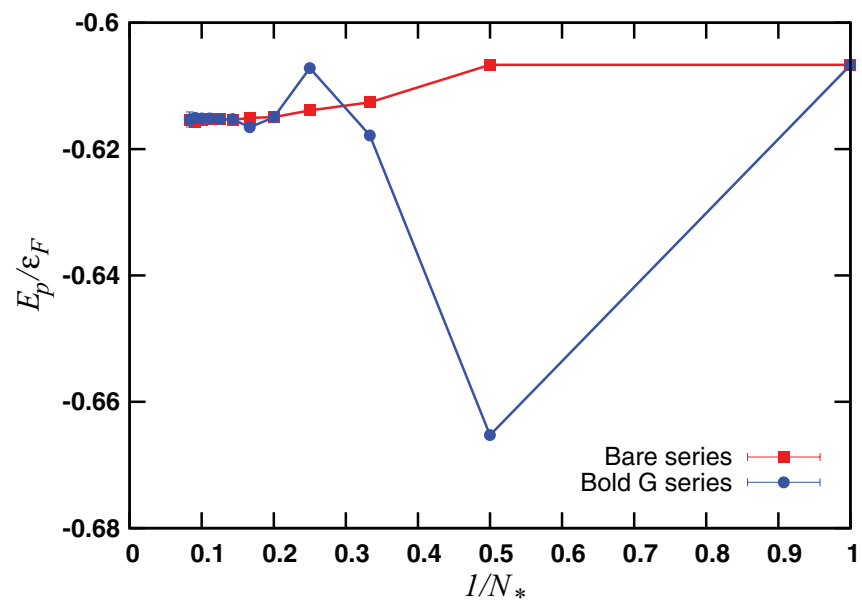

FIG. 5. (Color online) The polaron energy in units of the Fermi energy as a function of the inverse maximum diagram order $1 / N_{*}$ for irreducible self-energy diagrams at unitarity $1 /\left(k_{F} a\right)=0$. The (red) squares show the polaron energy calculated via Eq. (11) with self-energy diagrams built from the free propagators $G_{\sigma}^{0}$ and the partially dressed propagator $\Gamma^{0}$. The (blue) circles show the results using the bold $G$ approach. 


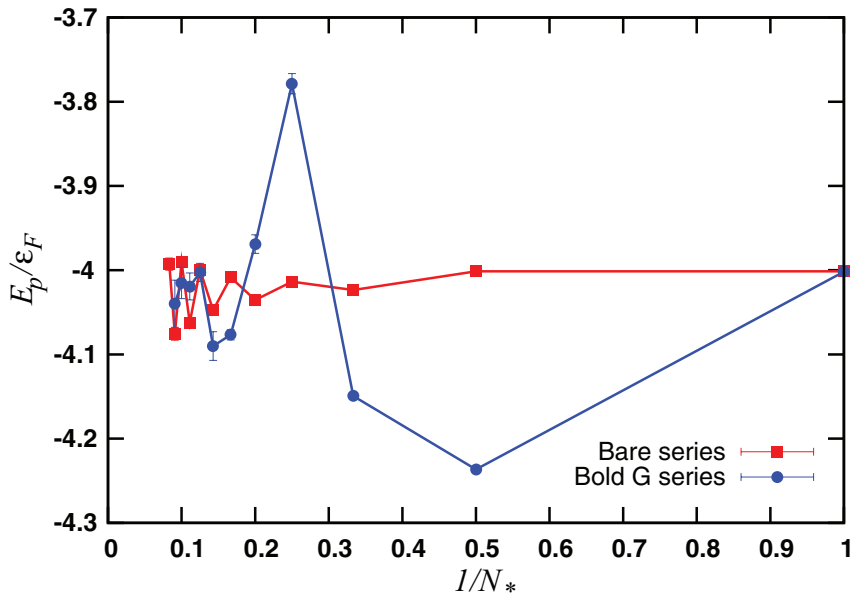

FIG. 6. (Color online) Same plot as Fig. 5, but now considering the interaction strength parameter $1 /\left(k_{F} a\right)=1.333$. In the bare series, small oscillations prevent us from extrapolating to the infinite diagram order.

order we have exactly the same type of cancellation between diagrams with the same number of hole propagators. ${ }^{16}$ Just as in the Combescot-Giraud argument, the cancellation is exact when the momentum dependence of the hole propagators is neglected. Note that the dominant diagrams (see Fig. 4) can also be viewed as three-body $T$-matrix diagrams closed with two-hole propagators. ${ }^{17}$ This class of diagrams, in which there are, at most, two particle-hole excitations, has been considered previously for the polaron problem. ${ }^{12,15}$ It was shown that they exactly reproduce the Skorniakov and Ter-Martirosian equation $^{18}$ in the BEC limit. In this strong-coupling limit, the dominant process is scattering between a dimer and a spin-up fermion, which is diagrammatically represented by the three-body $T$-matrix diagrams. Away from this limit, the considered class of diagrams turns out to give a quantitatively good correction to the lowest order result. We find that this is due to their dominance, even away from the BEC limit.

When going towards the BEC side $(1 / a>0)$, the cancellation between dominant diagrams of the type shown in Fig. 4 is no longer perfect. Figure 6 shows the polaron energy as a function of $1 / N_{*}$ for $1 /\left(k_{F} a\right)=1.333$. For the bare series, the oscillations prevent one from extracting $E_{p}$ for $1 / N_{*} \rightarrow 0$.

To cure the bad convergence of the bare series for $1 /\left(k_{F} a\right)>0$ one can include more diagrams by dressing the propagators. We start by dressing the spin-down propagator lines, while keeping the partially dressed $\Gamma^{0}$. Diagrams reducible with respect to cutting two spin-down lines should no longer be sampled, since they are included implicitly. For $1 /\left(k_{F} a\right)=0$ the self-energy $\Sigma(\mathbf{k}, \tau)$ converges in this "bold $G$ scheme" for $N_{*} \geqslant 7$. Extrapolation to infinite $N_{*}$ gives the exact $\Sigma$ and $G_{\downarrow}$. Figure 5 includes the polaron energy as a function of the diagram order cutoff when the one-body self-energy is built with the exact $G_{\downarrow}$. The bare and bold series converge to the same energy. Remarkably, the dressed scheme gives worse results at low $N_{*}$. This indicates that approximations based on a few low-order diagrams are completely uncontrolled, and including more diagrams by dressing the lines does not necessarily improve the quality of the results.

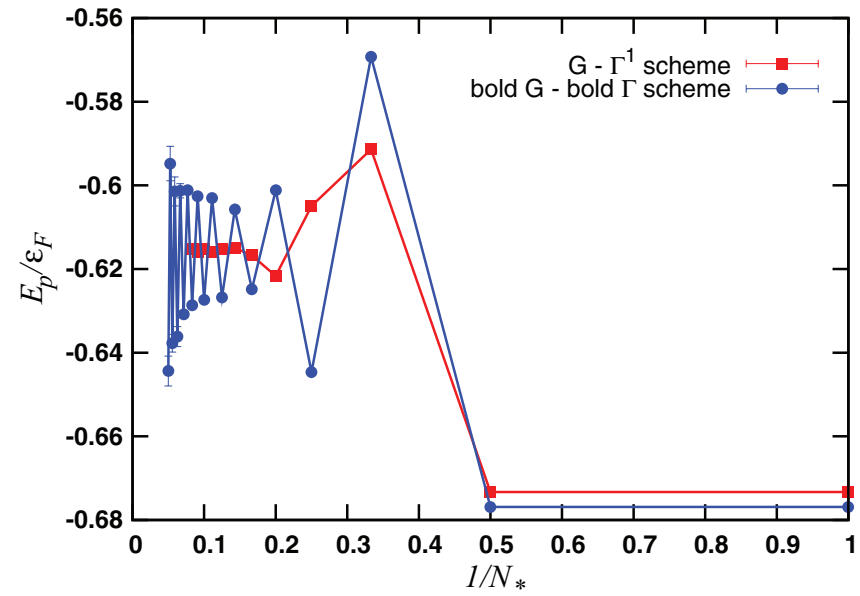

FIG. 7. (Color online) The polaron energy in units of the Fermi energy as a function of the inverse maximum diagram order $1 / N_{*}$ for irreducible self-energy diagrams at unitarity $1 /\left(k_{F} a\right)=0$. The (blue) circles show results using a fully bold DiagMC simulation: selfenergy diagrams are built from fully dressed one-particle propagators $G_{\downarrow}$ and two-particle propagators $\Gamma$ up to self-energy diagram order $N_{*}$. The (red) squares show the polaron energy calculated with diagrams built with the exact $G_{\downarrow}$ and a partially dressed interaction $\Gamma^{1}$ containing the sum of all $G_{\downarrow} G_{\uparrow}^{0}$ ladders.

For $1 /\left(k_{F} a\right)=1.333$, we see that dressing the impurity lines helps to get rid of the residual oscillations in the bare scheme (see Fig. 6). One might expect that dressing even more, by using a fully dressed $\Gamma$ instead of $\Gamma^{0}$, might lead to even better convergence. Figure 7 shows, however, that, even for $1 /\left(k_{F} a\right)=0$, the fully bold series (bold $G-\Gamma$ scheme) does not seem to converge ( $N_{*}$ is the diagram cutoff for both $\Sigma$ and $\Pi$, and a bold DiagMC simulation is done for each $N_{*}$ ), in contrast to the results in Ref. 8 . The data for the fully bold simulation in Ref. 8 was obtained by using the exact $G$ and $\Gamma$ (i.e., extrapolated to the $N_{*} \rightarrow \infty$ limit with resummation factors). They were not obtained with a self-consistent simulation, which explains the difference. Moreover, data are not shown above $N_{*}=7$, where oscillations do occur. In order to understand why the series no longer converges, we introduce an intermediate scheme (which we call bold $G-\Gamma^{1}$ ): the self-energy is built from the fully converged $G_{\downarrow}$ and a partially dressed interaction $\Gamma^{1}$, built from summing the ladders $G_{\downarrow} G_{\uparrow}^{0}$. The result is also shown in Fig. 7, and we again observe convergence to the same answer as in Fig. 5. The key difference between both schemes is that in the bold $G-\Gamma^{1}$ scheme, both dominant diagrams shown in Fig. 4 still explicitly contribute to the self-energy, whereas in the fully bold scheme the upper dominant diagram becomes reducible and is taken into account self-consistently. This means that the balance of cancellation between diagrams is broken, and a single dominant diagram keeps contributing at each order. So, it turns out that dressing the diagrams as much as possible is not always a good idea. In this respect, our findings disagree with Ref. 8.

The second method to cure the bad convergence of the bare series on the BEC side is to employ series resummation techniques. We use the Abelian resummation techniques ${ }^{19}$ which have been used for calculating the equation of state of a unitary gas with the bold DiagMC. ${ }^{20}$ This resummation 
technique works as follows. One starts from a series $f(x)=$ $\sum_{n} d_{n} x^{n}$ that has a finite radius of convergence $R>0$. The idea is to sum the series at some point $x_{0}$ outside of the radius $R$ by analytically continuing the function $f$. This provides a good procedure for summing the divergent series in the sense that it respects basic operations (sum, multiplication and derivative) and that it preserves distinctness. ${ }^{19}$ It is well known that with analytic continuation, one can encounter problems with the existence and/or uniqueness of the solution. ${ }^{21}$ However, one can formally define a domain called the "Mittag-Leffler star," where the function can be analytically continued along straight lines $\left[0, x_{0}\right]$. Note that this star will always contain the disk of convergence. It can be shown ${ }^{19}$ that, for each point $x_{0}$ of the Mittag-Leffler star, the limit

$$
\lim _{\epsilon \rightarrow 0^{+}} \sum_{n} d_{n} x_{0}^{n} e^{-\epsilon \lambda_{n}},
$$

with $\lambda_{n}=n \log (n)$ for $n>0$ and $\lambda_{0}=0$, exists and is equal to the analytic continuation of $f$ to the point $x_{0}$. Note that within the disk of convergence the procedure works equally well and can improve the rate of convergence. We apply the Abelian resummation technique for the expansion of the self-energy $\Sigma$ and $\Pi$. As the analytic structure of $\Sigma$ and $\Pi$ is unknown, it is currently impossible to determine whether there is a finite radius of convergence and whether we are in the Mittag-Leffler star. In practice, we apply different resummation techniques (i.e., different functions $\lambda_{n}$ ) and test the uniqueness of the result.

We use the following $\lambda_{n}$ : (i) Lindelöf $1, \lambda_{n}=n \log (n)$ for $n>0$ and $\lambda_{0}=0$; (ii) Lindelöf $2, \lambda_{n}=(n-1) \log (n-1)$ for $n>1$ and $\lambda_{0}=\lambda_{1}=0$; (iii) Gauss $1, \lambda_{n}=n^{2}$ for $n \geqslant 0$; (iv) Gauss 2, $\lambda_{n}=(n-1)^{2}$ for $n \geqslant 1$ and $\lambda_{0}=0$; and (iv) Gauss $3, \lambda_{n}=(n-2)^{2}$ for $n \geqslant 2$ and $\lambda_{0}=\lambda_{1}=0$. Before applying these resummation techniques to our diagrammatic series, we illustrate its power with an example for the geometric series. Figure 8 shows the sums $f_{\epsilon}\left(x_{0}=-3\right)=\sum_{n} x_{0}^{n} e^{-\epsilon \lambda_{n}}$ for a few choices of $\lambda_{n}$. At small $\epsilon$, the computation of $f_{\epsilon}$ is no longer feasible due to finite computer precision. By

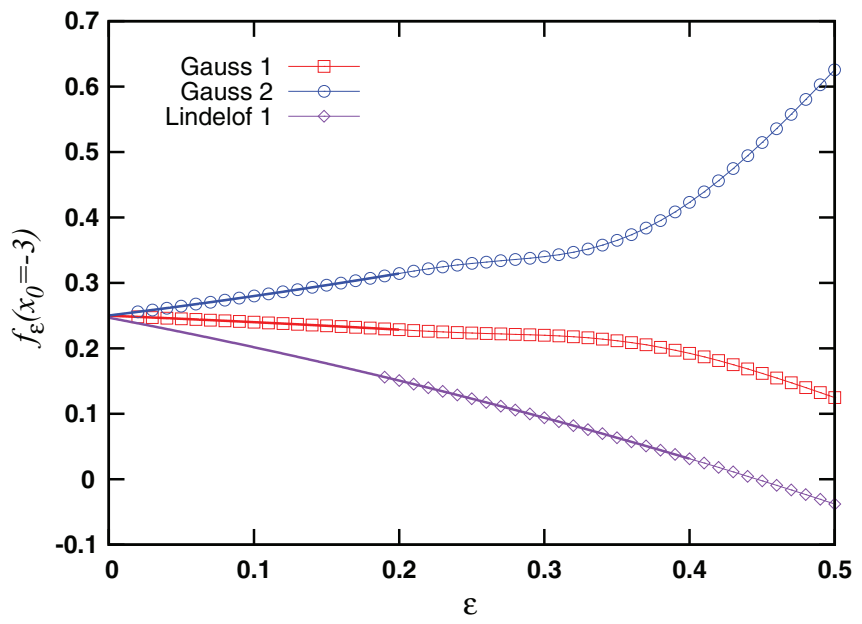

FIG. 8. (Color online) Illustration of the Abelian resummation technique for a geometric series. We evaluate $f_{\epsilon}\left(x_{0}\right)=\sum_{n} x_{0}^{n} e^{-\epsilon \lambda_{n}}$ for $x_{0}=-3$ and various choices of $\lambda_{n}$. The value of the analytically continued function $1 /\left(1-x_{0}\right)$ is retrieved for $\epsilon \rightarrow 0^{+}$.

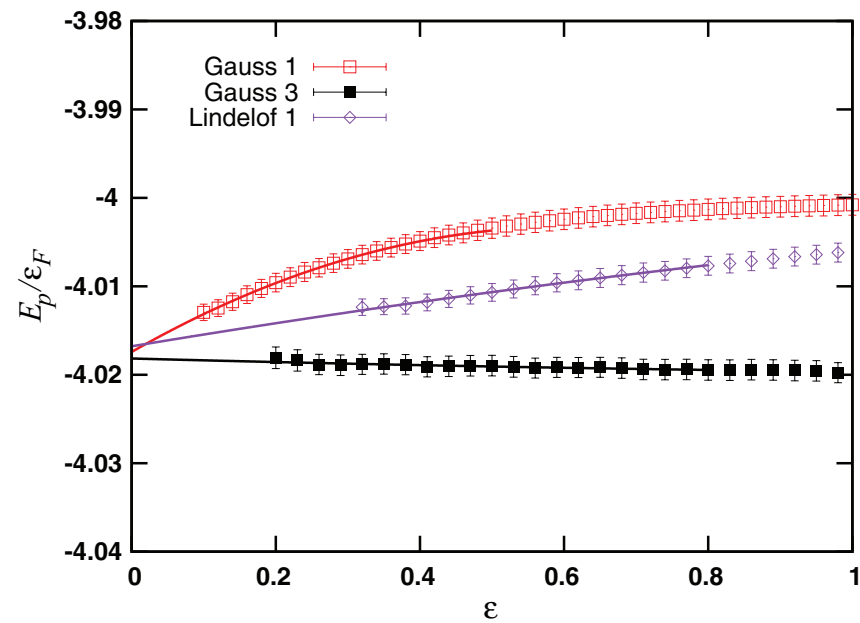

FIG. 9. (Color online) Abelian resummation of the bare series of one-body self-energy diagrams at $1 /\left(k_{F} a\right)=1.333$. The polaron energy $E_{p} / \epsilon_{F}$ is extracted in the limit $\epsilon=0^{+}$for different choices of $\lambda_{n}$.

extrapolating to $\epsilon=0$, we indeed find $1 /\left(1-x_{0}\right)$ with a high precision. The Lindelöf curve gives a slighly less accurate extrapolation because it suppresses high-order contributions in a much smoother fashion than the Gaussian resummation. When applying these techniques to our diagrammatic series, it is the growth of the statistical error bars (due to factorial complexity) that prevents us from going to very small values of $\epsilon$. Figure 9 shows the polaron energy calculated with the resummed self-energy as a function of the control parameter $\epsilon$ for $1 /\left(k_{F} a\right)=1.333$. The polaron energy $E_{p}$ can be extracted with a high accuracy. The major source of the error bar stems from the uncertainty in the extrapolation.

Histogramming the different topologies of the two-body self-energies $\Pi$ revealed a dominant diagram at each order. This diagram is shown in Fig. 10. Again, it shows a three-body $T$-matrix structure that is closed with a single spin-up hole propagator. Upon increasing the diagram order up to 20 , we observe a steady growth in the contribution of this diagram. This is illustrated in Fig. 11, where we plot the $n$ th-order contribution $\Pi_{n}$ to the two-body self-energy as a function of the imaginary time $\tau$ for external momentum 0 . Figure 12 illustrates that we can nonetheless get accurate values for the molecule energy $E_{\text {mol }}$ by using different Abelian resummation techniques and extrapolating to $\epsilon=0^{+}$. Again, the Gaussian resummation methods allow one to reach very small values of $\epsilon$. The quoted error bars are rather conservative, as we include the extrapolated results obtained with all choices for $\lambda_{n}$.

We also tested the resummability of the fully bold series (bold $G$-bold $\Gamma$ scheme), since this was used in the bold DiagMC method for determining the equation of state of the

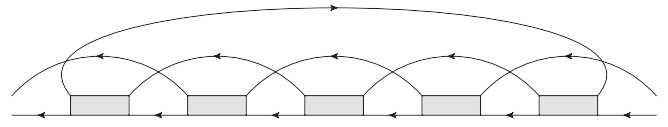

FIG. 10. At fixed order, there is one dominant diagram for the two-body self-energy. Here, we draw this diagram at order 6. 


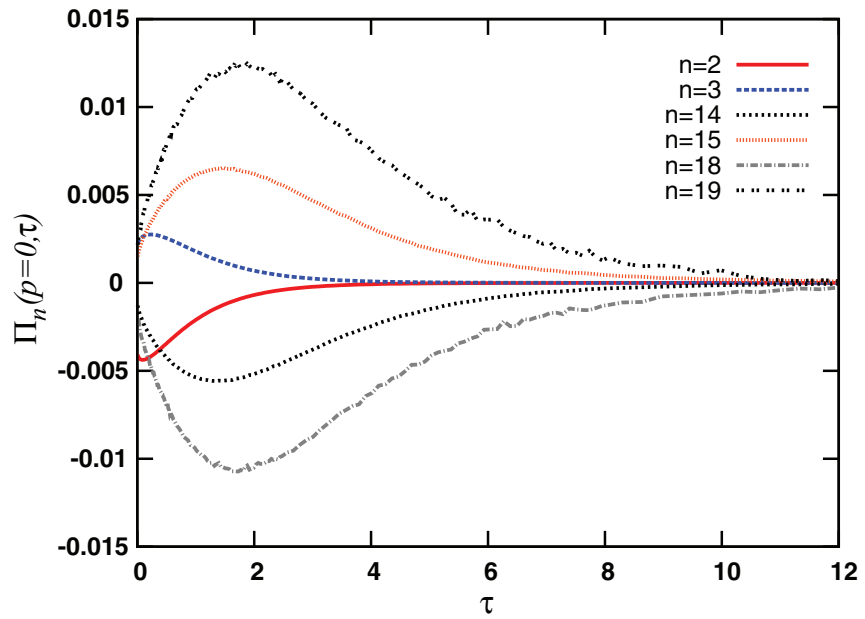

FIG. 11. (Color online) The two-particle self-energy $\Pi$ at external momentum 0 as a function of imaginary time for $k_{F} a=1 . \Pi_{n}$ values are the contributions of all $n$ th-order diagrams and are shown for various $n$. As $n$ increases, $\Pi_{n}$ keeps on growing. We work in units $k_{F}=1, m=1, \hbar=1$, and $\mu / \epsilon_{F}=-3.2$. The noise in the curves indicates the magnitude of the statistical error.

unitary gas. ${ }^{20}$ Upon application of the Abelian resummation techniques and extrapolation to $\epsilon=0^{+}$at $1 /\left(k_{F} a\right)=0$, the correct polaron energy is retrieved. This constitutes an independent check for the resummation of the skeleton series.

\section{QUASIPARTICLE PROPERTIES}

As an independent cross-check of Ref. 3, which uses alternate ways of resumming the diagrammatic series, we calculate the ground-state energies of the polaron and molecule. Figure 13 shows these energies shifted by the vacuum molecule energy $E_{b}=-1 /\left(m a^{2}\right)$ in units of the Fermi energy $\epsilon_{F}$. A selection of the polaron and molecule energies is also reported in Table II. We find the transition point at $\left(k_{F} a\right)_{c}=1.15(3)$, in agreement with Ref. 3. Close to the transition point, we find polaron energies that differ about $1 \%$ from the polaron

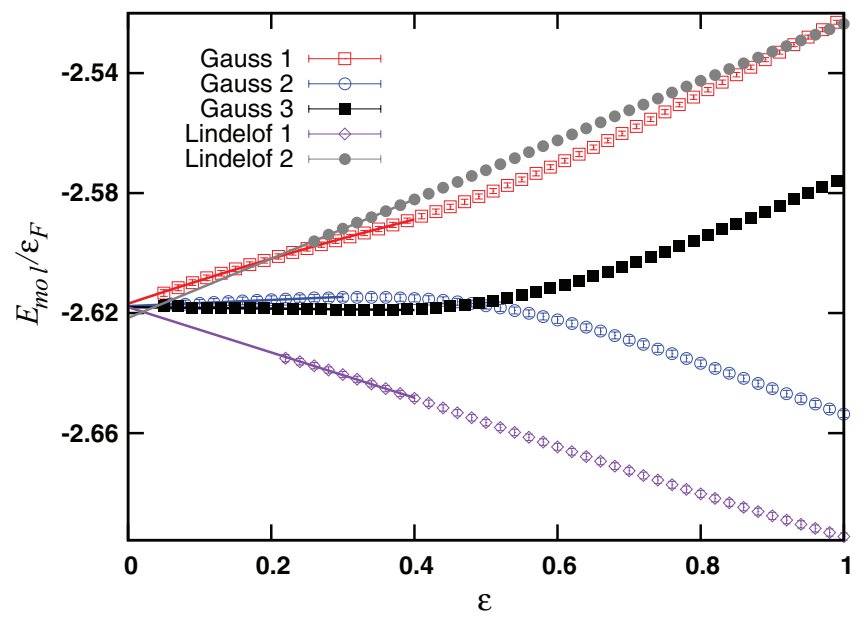

FIG. 12. (Color online) Abelian resummation of the bare series of two-particle self-energy diagrams at $k_{F} a=1$. The molecule energy $E_{\mathrm{mol}} / \epsilon_{F}$ is extracted in the limit $\epsilon=0^{+}$for different choices of $\lambda_{n}$.

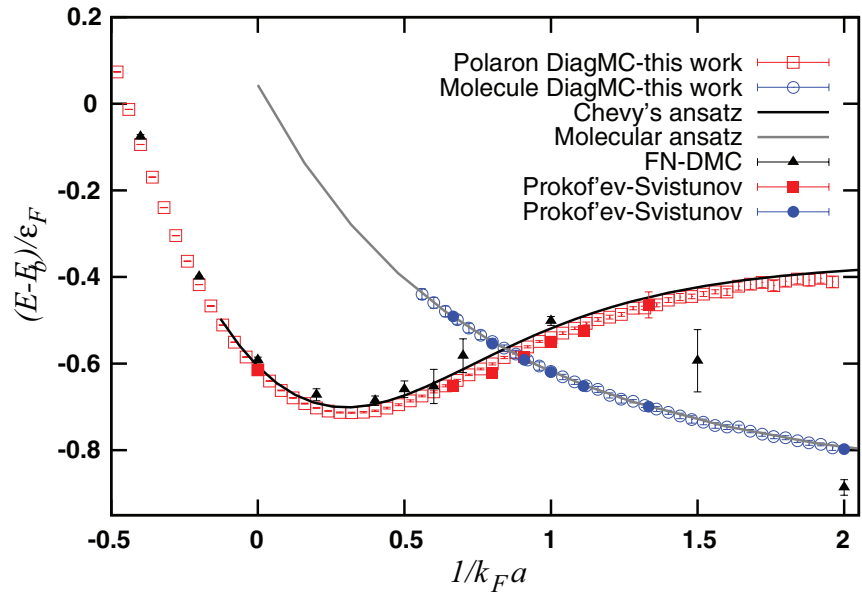

FIG. 13. (Color online) The extracted polaron and molecule energies as a function of the interaction strength $1 /\left(k_{F} a\right)$. Energies are expressed as $\left(E-E_{b}\right) / \epsilon_{F}$, with $E_{b}=-1 /\left(m a^{2}\right)$ the molecule energy in vacuum. FN-DMC results are from Ref. 6; variational results for the polaron, from Ref. 9; and variational results for the molecule, from Ref. 11. DiagMC results of Prokof'ev and Svistunov ${ }^{3}$ are also shown.

energies in Ref. 3, which, we believe, is due to a small systematic error in the lowest order diagram in Ref. 3. The variational energies obtained from a wave-function ansatz for the polaron ${ }^{9}$ and the molecule ${ }^{11}$ are very close to the MC results. Note that Chevy's variational ansatz for the polaron state is completely equivalent with the non-self-consistent $T$-matrix approximation, ${ }^{12}$ which is exactly our bare series at $N_{*}=1$. Fixed node-diffusion MC (FN-DMC) results are also in good agreement with the DiagMC data. For $1 /\left(k_{F} a\right)=2$ it seems that systematic errors in the FN-DMC results were

TABLE II. Selection of DiagMC data for the polaron energy $E_{p}$, molecule energy $E_{\mathrm{mol}}$, and polaron residue $Z_{p}$ for several values of the interaction strength parameter $1 /\left(k_{F} a\right)$.

\begin{tabular}{lrrr}
\hline \hline $1 /\left(k_{F} a\right)$ & $E_{p} / E_{F}$ & $E_{\mathrm{mol}} / E_{F}$ & $Z_{p}$ \\
\hline-1.8 & $-0.1793(1)$ & & $0.9727(4)$ \\
-1.6 & $-0.1961(1)$ & & $0.9665(5)$ \\
-1.4 & $-0.2159(2)$ & & $0.9590(3)$ \\
-1.2 & $-0.2393(2)$ & & $0.9502(3)$ \\
-1.0 & $-0.2687(2)$ & & $0.9376(4)$ \\
-0.8 & $-0.3052(2)$ & & $0.9209(5)$ \\
-0.6 & $-0.3526(2)$ & & $0.8978(8)$ \\
-0.4 & $-0.4141(2)$ & & $0.8670(10)$ \\
-0.2 & $-0.4976(2)$ & & $0.8237(15)$ \\
0.0 & $-0.615(1)$ & & $0.7586(27)$ \\
0.2 & $-0.782(1)$ & & $0.6720(42)$ \\
0.4 & $-1.028(2)$ & & $0.5672(28)$ \\
0.6 & $-1.385(2)$ & $-1.180(13)$ & $0.4410(32)$ \\
0.8 & $-1.880(2)$ & $-1.830(8)$ & $0.3258(58)$ \\
1.0 & $-2.540(3)$ & $-2.618(6)$ & $0.2283(70)$ \\
1.2 & $-3.372(4)$ & $-3.554(6)$ & $0.1559(69)$ \\
1.4 & $-4.373(5)$ & $-4.633(5)$ & $0.1102(68)$ \\
1.6 & $-5.554(8)$ & $-5.867(6)$ & $0.0771(58)$ \\
1.8 & $-6.889(12)$ & $-7.251(5)$ & $0.0578(35)$ \\
\hline \hline
\end{tabular}




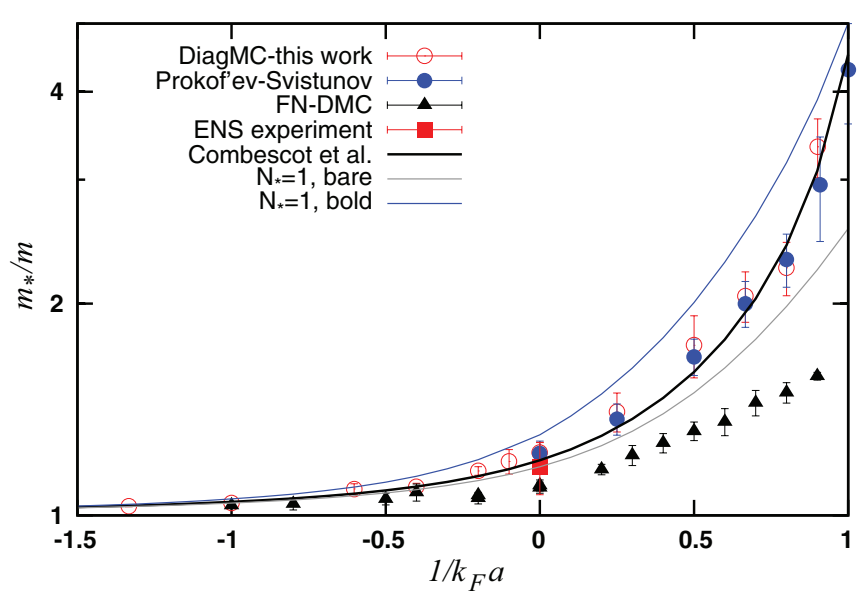

FIG. 14. (Color online) The effective mass $m_{*}$ of the polaron in units of the bare mass $m$ as a function of the interaction parameter $1 /\left(k_{F} a\right)$. Our DiagMC results (open circles) are shown together with DiagMC results of Prokof'ev and Svistunov ${ }^{3}$ [filled (blue) circles], FN-DMC results ${ }^{6,7}$ [filled (black) triangles], ENS experiment ${ }^{5}$ [filled (red) square], and a variational calculation up to two particle-hole excitations $^{12}$ (solid black line). We also show $m_{*}$ values calculated from the lowest order self-energy diagram (i.e., $N_{*}=1$ ) for the bare series (solid gray line) and for the fully bold $G-\Gamma$ series (solid blue line), which are equivalent to the non-self-consistent and the selfconsistent $T$-matrix approximations, respectively.

underestimated, since the FN-DMC should, in principle, give an upper bound to the true ground-state energy.

Figure 14 shows the effective mass of the polaron as calculated with the DiagMC. We compare it with the ENS experiment ${ }^{5}$ at unitarity, the DiagMC calculations by Prokof'ev and Svistunov, ${ }^{3}$ the FN-DMC, ${ }^{6,7}$ a variational calculation up to two particle-hole excitations, ${ }^{12}$ and the first-order $\left(N_{*}=1\right)$ result in the bare scheme and the fully bold $G-\Gamma$ scheme. The experimental effective mass, which is in perfect agreement with DiagMC, ${ }^{3}$ was extracted from the low-frequency breathing modes, in particular, the Fermi polaron breathing mode. The lowest order bare calculation, also known as the $T$-matrix approximation, is equivalent to the Chevy ansatz, while the lowest order bold calculation corresponds to the self-consistent $T$-matrix approximation. These results show that including only single particle-hole pair excitations does not lead to accurate results for the effective mass, while the variational calculation based on diagrams taking into account at most two particle-hole pairs excitations agrees with the DiagMC results. ${ }^{12}$

Experimental and theoretical quasiparticle residues are shown in Fig. 15. To create and probe polarons, the MIT experiment ${ }^{4}$ starts from a cloud of ${ }^{6} \mathrm{Li}$ atoms, with most atoms occupying the lowest hyperfine state $|1\rangle$ (spin-up) and about $2 \%$ of the atoms occupying the hyperfine state $|3\rangle$ (spin-down) in the degenerate regime $T \approx 0.14 T_{F}$, with $T_{F}$ the Fermi temperature. A broad Feshbach resonance is used to enhance the scattering between atoms in state $|1\rangle$ and those in state $|3\rangle$. The radio-frequency spectra of the spin-up and spin-down components are measured. The atoms are transferred to a third empty state with very weak final-state interactions. Therefore, the measured transition rate $I$ can be connected with the

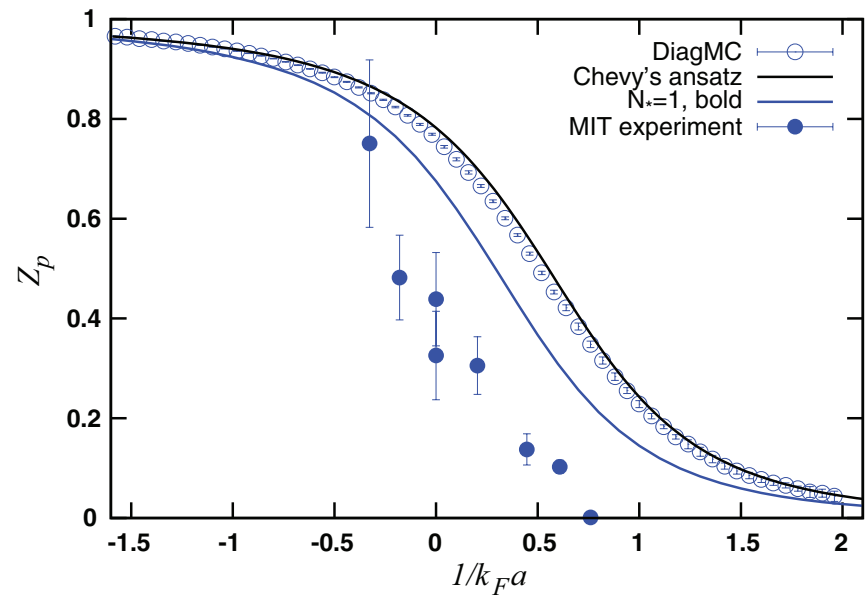

FIG. 15. (Color online) The polaron quasiparticle residue $Z_{p}$ as a function of the interaction parameter $1 /\left(k_{F} a\right)$. DiagMC results (open circles) are compared with variational ansatz ${ }^{11}$ (solid black line), the fully bold $G-\Gamma$ series at $N_{*}=1$ or the self-consistent $T$-matrix approximation (solid blue line), and the MIT experiment ${ }^{4}$ [filled (blue) circles].

impurity's spectral function $\rho_{\downarrow}$ in linear response theory, ${ }^{22,23}$

$$
I\left(\omega_{L}\right) \propto \sum_{\mathbf{k}} n_{F}\left(\epsilon_{\mathbf{k}}-\mu-\omega_{L}\right) \rho_{\downarrow}\left(\mathbf{k}, \epsilon_{\mathbf{k}}-\mu-\omega_{L}\right),
$$

with $\omega_{L}$ the frequency of the radio-frequency photons and $n_{F}(x)=1 /\left(1+e^{\beta x}\right)$ the Fermi distribution. Note that the spectral function depends on the temperature. Density inhomogeneities are taken care of through tomographic reconstruction. ${ }^{4}$ At sufficiently weak attractions, the Fermi polaron is observed as a narrow peak in the impurity spectrum that is not matched by the broad environment spectrum. The peak position gives the polaron energy $E_{p}$ and was found to be in perfect agreement with the DiagMC results in Ref. 3 . The polaron $Z$ factor was measured by determining the ratio of the area under the impurity peak that is not matched by the environment to the total area under the impurity's spectrum. The experimental $Z$ factor from Ref. 4 is shown in Fig. 15, together with the $Z$ factor calculated from Chevy's ansatz, ${ }^{11,24}$ the fully self-consistent result to lowest order $\left(N_{*}=1\right)$, and our DiagMC simulation. DiagMC data for the $Z$ factor are also listed in Table II.

The results obtained via DiagMC simulation agree extremely well with Chevy's variational ansatz. This is very surprising in the strongly interacting regime where $Z_{p}$ is significantly less than 1 . Here, one would expect multiple particle-hole excitations to be important since the overlap with the noninteracting wave function is small. Remarkably, including just single particle-hole excitations on top of the FS produces almost the exact $Z_{p}$. When the lowest order diagram is calculated in a fully self-consistent way, however, the agreement with DiagMC is less good. This hints at the fact that the almost-perfect agreement with Chevy's ansatz (i.e., the lowest order bare result) is rather accidental. The $Z$ factors computed with Chevy's ansatz and the DiagMC both exceed the measured ones.

It was suggested in Ref. 11 that the disagreement between the experiment and the Chevy ansatz is an artifact of 
Chevy's expansion being restricted to one-particle/one-hole excitations. As the DiagMC technique includes multipleparticle/multiple-hole excitations and agrees very well with Chevy's ansatz, we see that this is not the case. However, since the measured $Z_{p}$ might only give a lower bound, ${ }^{4}$ theory and experiment might not be in disagreement.

The measured polaron $Z$ factor vanishes beyond a critical interaction strength. Ignoring issues related to metastability, once the two-body bound state becomes energetically favorable, all polarons disappear and the polaron peak vanishes. In the experiment $T / T_{F}=0.14(3)$, and finite-temperature effects are thus expected to become important. Indeed, close to $\left(k_{F} a\right)_{c}=1.15(3)$ the energy difference between the molecule and the polaron state is of the order of $0.1 T_{F}$ (Fig. 13). Therefore, one expects that $T=0$ calculations underestimate the critical $1 /\left(k_{F} a\right)$ measured at $T \approx 0.1 T_{F}$. Similarly, the measured $1 /\left(k_{F} a\right)_{c}$ can be interpreted as an upper bound for the $T=0$ situation. On the other hand, due to depletion of the experimental spectrum, the measured $Z$ might only give a lower bound, which means that the experimentally determined critical $1 /\left(k_{F} a\right)$ might be underestimated. These uncertainties might explain why the critical $1 /\left(k_{F} a\right)$ in the experiment is lower than the value obtained with DiagMC for a single impurity. Fixed-node MC simulations for a finite density of impurities, on the other hand, predict phase separation before the systems even reaches the polaron-to-molecule transition, ${ }^{6}$ and the vanishing $Z$ factor might be a manifestation of this phase separation.

\section{CONCLUSIONS}

We have considered the Fermi-polaron system in three dimensions, in which a single spin-down impurity is strongly coupled to a noninteracting FS of spin-up particles. Although this system contains strongly interacting fermions, it can be solved with the DiagMC method. This method is based on the stochastic evaluation of a series of Feynman diagrams. To extract ground-state properties, one has to overcome a factorial complexity due to the increase in the number of diagrams. Nonetheless, extrapolation to infinite diagram order becomes possible when the diagrams cancel each other better than the factorial increase in number. At interaction strength $1 /\left(k_{F} a\right)=$ 0 , we find such perfect cancellation (within our statistical errors). When considering the series built on bare propagators on the BEC side, however, oscillations with diagram order remain and prevent a controlled extrapolation to the infinite diagram order. We have followed two strategies around this problem: the first is to consider skeleton series (built on dressed propagators); the second, to use resummation techniques. Though dressed series can be evaluated to higher orders, we have found that in some cases dressing can destroy a favorable cancellation of diagrams. For all interaction strengths we found that the (skeleton) series of the one-body and two-body selfenergies are resummable by means of Abelian resummation. The bare series, skeleton series, and resummed series give robust answers in their respective regions of applicability (i.e., where the infinite-diagram-order extrapolation is controlled).

We have identified classes of dominant diagrams for the one-body and two-body self-energy in the crossover region of strong interaction. The dominant diagrams turn out to be the leading processes of the strong-coupling limit: scattering between a dimer and a spin-up fermion, which is diagrammatically represented by the three-body $T$-matrix diagrams. Including just these dominant diagrams gives a quantitatively good correction to the lowest order result, even away from the strong-coupling limit.

We have shown that not only do the polaron and molecule energies agree very well with a variational ansatz from weak to strong attraction, but also the polaron residue or $Z$ factor. Though this agreement must be due to strong cancellation of diagrams, we only observed convergence for the bare series at $1 /\left(k_{F} a\right)=0$. A full explanation of the success of the variational ansatz is still missing, and it is therefore unclear in which cases the ansatz is appropriate.

\section{ACKNOWLEDGMENTS}

The authors would like to thank C. Lobo, N. Prokof'ev, B. Svistunov, F. Werner, and M. Zwierlein for helpful discussions and suggestions. We thank R. Combescot, S. Pilati, N. Prokof'ev, M. Punk, B. Svistunov, and M. Zwierlein for sending us their data. This work was supported by the Fund for Scientific Research-Flanders.
*Corresponding author: kris.vanhoucke@ugent.be

${ }^{1}$ L. D. Landau, Phys. Z. Sowjetunion 3, 664 (1933).

${ }^{2}$ E. M. Lifshitz and L. P. Pitaevskii, Statistical Mechanics (Pergamon Press, New York, 1980), Part 2.

${ }^{3}$ N. V. Prokof'ev and B. V. Svistunov, Phys. Rev. B 77, 020408 (2008).

${ }^{4}$ A. Schirotzek, C. H. Wu, A. Sommer, and M. W. Zwierlein, Phys. Rev. Lett. 102, 230402 (2009).

${ }^{5}$ S. Nascimbène, N. Navon, K. J. Jiang, L. Tarruell, M. Teichmann, J. McKeever, F. Chevy, and C. Salomon, Phys. Rev. Lett. 103, 170402 (2009).

${ }^{6}$ S. Pilati and S. Giorgini, Phys. Rev. Lett. 100, 030401 (2008).
${ }^{7}$ S. Pilati (private communication).

${ }^{8}$ N. V. Prokof'ev and B. V. Svistunov, Phys. Rev. B 77, 125101 (2008).

${ }^{9}$ F. Chevy, Phys. Rev. A 74, 063628 (2006).

${ }^{10}$ C. Mora and F. Chevy, Phys. Rev. A 80, 033607 (2009).

${ }^{11}$ M. Punk, P. T. Dumitrescu, and W. Zwerger, Phys. Rev. A 80, 053605 (2009).

${ }^{12}$ R. Combescot, S. Giraud, and X. Leyronas, Europhys. Lett. 88 (2009) 60007.

${ }^{13}$ N. V. Prokof'ev and B. V. Svistunov, Phys. Rev. Lett. 99, 250201 (2007).

${ }^{14}$ N. M. Hugenholtz, Physica 23, 533 (1957). 
${ }^{15}$ R. Combescot and S. Giraud, Phys. Rev. Lett. 101, 050404 (2008).

${ }^{16}$ S. Giraud, Ph.D. thesis, Université Paris VI (2010); http://tel.archives-ouvertes.fr/tel-00492339.

${ }^{17}$ X. Leyronas and R. Combescot, Phys. Rev. Lett. 99, 170402 (2007).

${ }^{18}$ G. V. Skorniakov and K. A. Ter-Martirosian, Zh. Eksp. Teor. Fiz. 31, 775 (1956) [Sov. Phys. JETP 4, 648 (1957)].

${ }^{19}$ G. H. Hardy, Divergent Series (Oxford University Press, New York, 1956).
${ }^{20}$ K. Van Houcke, F. Werner, E. Kozik, N. Prokofev, B. Svistunov, M. J. H. Ku, A. T. Sommer, L. W. Cheuk, A. Schirotzek, and M. W. Zwierlein, Nature Phys. 8, 366 (2012).

${ }^{21}$ P. Dennery and A. Krzywicki, Mathematics for Physicists (Dover, New York, 1996).

${ }^{22}$ Y. Ohashi and A. Griffin, Phys. Rev. A 72, 013601 (2005).

${ }^{23}$ P. Massignan, G. M. Bruun, and H. T. C. Stoof, Phys. Rev. A 77, 031601(R) (2008).

${ }^{24}$ C. Trefzger and Y. Castin, Europhys. Lett. 101, 30006 (2013). 